\title{
THREE COUNTEREXAMPLES FOR A QUESTION CONCERNING GREEN'S FUNCTIONS AND CIRCULAR SYMMETRIZATION
}

\author{
ALEXANDER R. PRUSS
}

(Communicated by Albert Baernstein II)

\begin{abstract}
We construct domains $U$ in the plane such that if $G\left(r e^{i \theta}\right)$ is the Green's function of $U$ with pole at zero, while $\tilde{G}\left(r e^{i \theta}\right)$ is the symmetric non-increasing rearrangement of $G\left(r e^{i \theta}\right)$ for each fixed $r$ and $G^{*}$ is the Green's function of the circular symmetrization $U^{*}$, again with pole at zero, then there are positive numbers $r$ and $\varepsilon$ such that

$$
G^{*}\left(r e^{i \theta}\right)<\tilde{G}\left(r e^{i \theta}\right)
$$

whenever $0<|\pi-\theta|<\varepsilon$. One of our constructions will have $U$ simply connected. We also consider the case where the poles of the Green's functions do not lie at the origin. Our work provides a negative answer to a question of Hayman (1967).
\end{abstract}

\section{INTRODUCTION}

Given a domain $U$ in the plane, for fixed $r$, if $\{|z|=r\} \subset U$, then let $\theta(r ; U)=\infty$; otherwise, let $\theta(r ; U)=\left|\left\{\theta \in[0,2 \pi): r e^{i \theta} \in U\right\}\right|$. Now set $U^{*}=\left\{r e^{i \theta}:|\theta|<\right.$ $\theta(r ; U) / 2\}$. We then have $\theta\left(r ; U^{*}\right)=\theta(r ; U)$, and call $U^{*}$ the circular symmetrization of $U$. A domain $U$ is said to be circularly symmetric if $U=U^{*}$. See the figures in the text, below, for some examples.

Let $g(\cdot, w ; U)$ be the Green's function of $U$ with pole at some fixed point $w \in U$. This will be a non-negative function in $U$, harmonic away from $w$, with a logarithmic pole at $w$ (so that $g(z ; U)-\log \frac{1}{|z-w|}$ is harmonic near $z=w$ ), which vanishes outside $U$. Let

$$
U_{w, \lambda}=\{z \in U: g(z, w ; U)>\lambda\} .
$$

Then, $U_{w, \lambda}$ is circularly symmetric for every $\lambda \geq 0$ if and only if $U$ is circularly symmetric and $w \geq 0$ (one implication follows from the fact that $U_{0}=U$; the other is due to Baernstein [1, Corollary on p. 154]). Hayman [3, Question 5.17] had asked whether we necessarily have $\left(U_{w, \lambda}\right)^{*} \subseteq\left(U^{*}\right)_{|w|, \lambda}$. As Baernstein [1] notes, this is the same as asking whether we always have

$$
g\left(r e^{i \theta},|w| ; U^{*}\right) \geq \tilde{g}\left(r e^{i \theta}, w ; U\right),
$$

Received by the editors September 30, 1994 and, in revised form, November 16, 1994.

1991 Mathematics Subject Classification. Primary 31A15.

Key words and phrases. Green's functions, circular symmetrization.

The research was partially supported by Professor J. J. F. Fournier's NSERC Grant \#4822. This paper constitutes a portion of the author's doctoral dissertation. 


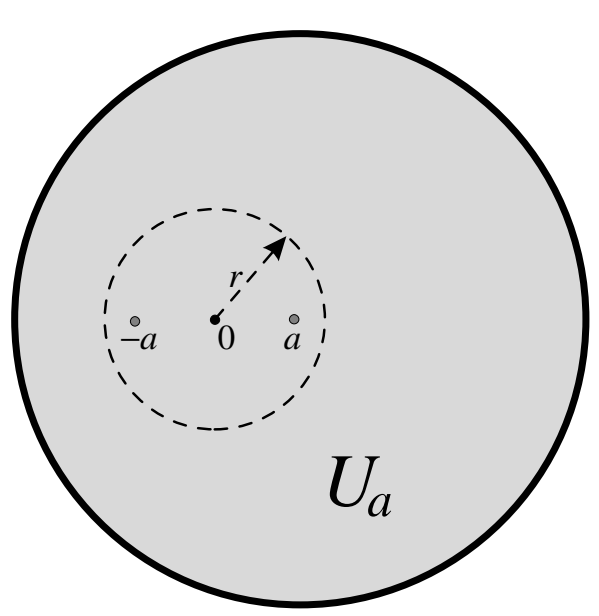

FiguRE 1 . The circularly symmetric domain $U_{a}$. The pole will be at $a$ or at $-a$.

where $\tilde{g}\left(r e^{i \theta}, w ; U\right)$ is the circularly symmetric non-increasing rearrangement of $e^{i \theta} \mapsto g\left(r e^{i \theta}, w ; U\right)$. This circularly symmetric non-increasing rearrangement is, for fixed $r$, a function $\theta \mapsto \tilde{g}\left(r e^{i \theta}, w ; U\right)$ on $[-\pi, \pi]$ which is symmetric about $\theta=0$, non-increasing and right-hand continuous with respect to $|\theta|$, and equimeasurable with the function $\theta \mapsto g\left(r e^{i \theta}, w ; U\right)$. The equivalence then follows from the fact that $\left\{z \in U: \tilde{g}\left(r e^{i \theta}, w ; U\right)>\lambda\right\}=\left(U_{w, \lambda}\right)^{*}$. Baernstein [1] had proved the weaker inequality that

$$
\int_{0}^{\theta_{1}} g\left(r e^{i \theta},|w| ; U^{*}\right) d \theta \geq \int_{0}^{\theta_{1}} \tilde{g}\left(r e^{i \theta}, w ; U\right) d \theta,
$$

for $0 \leq \theta_{1} \leq \pi$. However, we will show that in general the stronger inequality (1) is not valid, and the answer to Hayman's question is negative, even when restricted to $U$ being simply connected and $w=0$. In one of our examples, (1) will be false even though $U$ is circularly symmetric (but of course $w$ cannot lie on the non-negative real axis then).

\section{Counterexamples}

We give three counterexamples. The first is the easiest, and this is the one with $U$ circularly symmetric. Fix any $0<a<\frac{1}{2}$. Let $U_{a}$ be a disc of unit radius centered on the point $a$. Clearly, $U_{a}$ is circularly symmetric and $U_{a}^{*}=U_{a}$. (See Figure 1.)

Theorem 1. There exists $r_{1} \in(a, 1-a)$ such that for any $r \in\left(a, r_{1}\right]$ we have

$$
\min _{\theta} g\left(r e^{i \theta}, a ; U_{a}\right)=g\left(-r, a ; U_{a}\right)<\min _{\theta} g\left(r e^{i \theta},-a ; U_{a}\right) .
$$

The completely elementary proof will be given later. This gives a counterexample

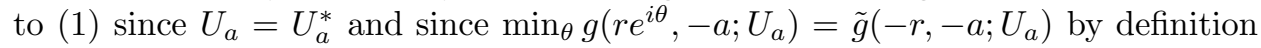
of $\tilde{g}$.

We now restrict the pole to lie at zero. This will make things a little more difficult. 

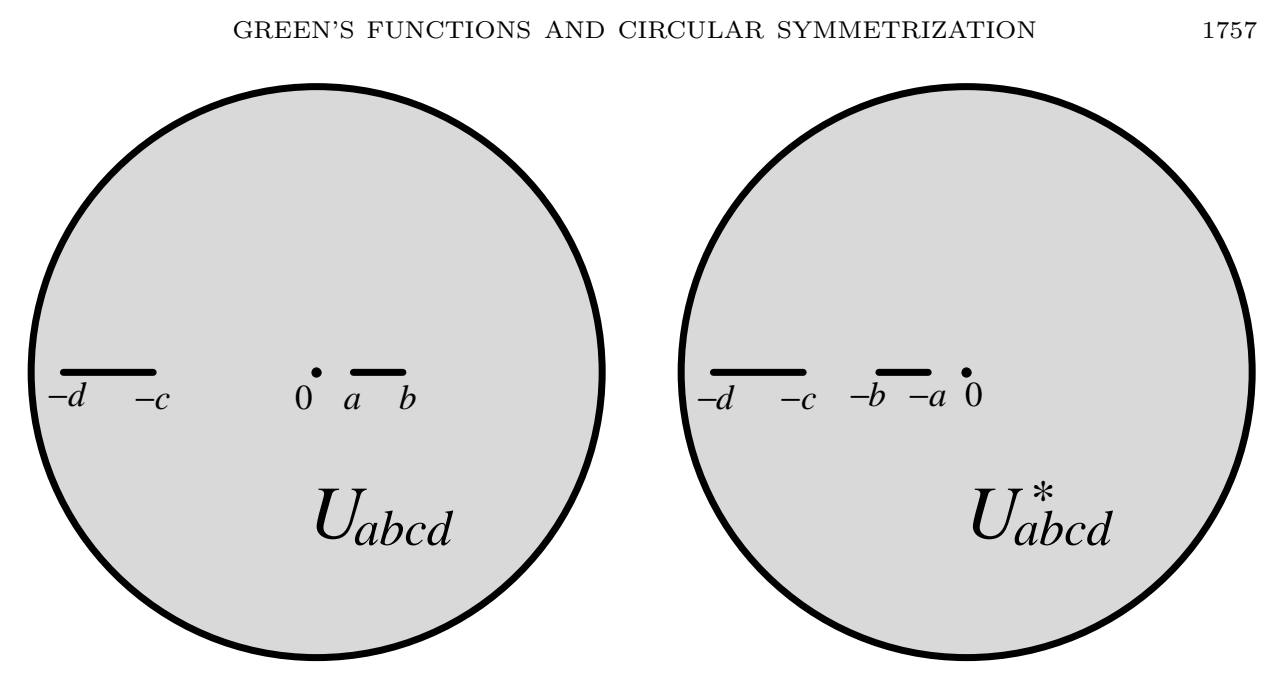

FiguRE 2. The unsymmetrized domain $U_{a b c d}$ and the symmetrized domain $U_{a b c d}^{*}$. The poles will be at the origin.

Theorem 2. There exist a domain $U$ in the plane and positive numbers $r$ and $\varepsilon$ such that

$$
g\left(r e^{i \theta}, 0 ; U^{*}\right)<\tilde{g}\left(r e^{i \theta}, 0 ; U\right),
$$

whenever $0<|\pi-\theta|<\varepsilon$. Moreover, $U$ may be taken to be simply connected.

This is of course also a counterexample to (1). We shall present two such counterexamples, one simply connected and one not, because the two examples have rather interesting and different proofs.

The multiply connected example is constructed as follows. Fix $0<a<b \leq$ $c<d \leq 1$. Let $U_{a b c d}=D \backslash([-d,-c] \cup[a, b])$, where $D$ is the unit disc. Clearly $U_{a b c d}^{*}=D \backslash([-d,-c] \cup[-b,-a])$. (See Figure 2.)

Lemma 1. There exist $r \in(a, b) \cup(c, d)$ and $\varepsilon>0$ such that

$$
g\left(r e^{i \theta}, 0 ; U_{a b c d}^{*}\right)<\tilde{g}\left(r e^{i \theta}, 0 ; U_{a b c d}\right),
$$

whenever $0<|\pi-\theta|<\varepsilon$.

The simply connected example is constructed as follows. Fix $0<a<b<1$. Let $V_{b}=\{z:|z|<1, \operatorname{Re} z>-b\}$ be a disc with a piece sliced off, and let $U_{a b}$ be $V_{b}$ slit along the positive real axis starting at $a$, namely

$$
U_{a b}=V_{b} \backslash[a, 1) .
$$

Then clearly $U_{a b}$ is simply connected and $U_{a b}^{*}=V_{b} \backslash(-b,-a]$. (See Figure 3, ignoring the cone $C_{-b, \delta} \subset U_{a b}^{*}$ for now.)

Lemma 2. There exists $a^{\prime} \in(a, b)$ with the property that for every $r \in\left[a^{\prime}, b\right)$ there is an $\varepsilon>0$ such that

$$
g\left(r e^{i \theta}, 0 ; U_{a b}^{*}\right)<\tilde{g}\left(r e^{i \theta}, 0 ; U_{a b}\right),
$$

whenever $0<|\pi-\theta|<\varepsilon$. 

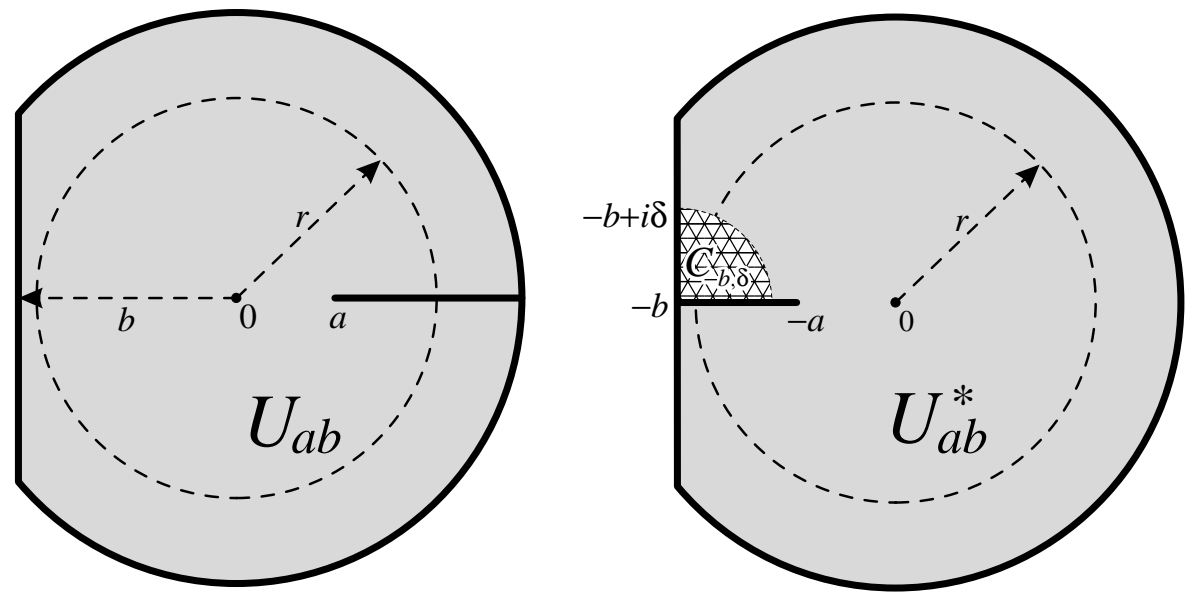

FiguRE 3. The unsymmetrized domain $U_{a b}$ and the symmetrized domain $U_{a b}^{*}$, together with the cone $C_{-b, \delta} \subset U_{a b}^{*}$ used in the proof. The poles will be at the origin.

\section{Proofs}

Proof of Theorem 1. Let $\mathfrak{g}(\cdot, w)$ be Green's function for the unit disc with a pole at $w$, so that

$$
\mathfrak{g}(z, w)=\log \left|\frac{1-z \bar{w}}{z-w}\right| .
$$

Since $1-a>\frac{1}{2}>a$, we need only work with $0 \leq r<1-a$. Write $U=U_{a}=U_{a}^{*}$. Then, $g\left(r e^{i \theta},-a ; U\right)=\mathfrak{g}\left(r e^{i \theta}-a,-2 a\right)$ and $g\left(r e^{i \theta}, a ; U\right)=\mathfrak{g}\left(r e^{i \theta}-a, 0\right)$. We can see directly that

$$
\min _{\theta} g\left(r e^{i \theta}, a ; U\right)=\mathfrak{g}(-r-a, 0)=\log \frac{1}{|r+a|} .
$$

Note that in fact in general if $V$ is a circularly symmetric domain and $v$ is on the positive real axis, then $g\left(r e^{i \theta}, v ; V\right)$ is symmetrically decreasing with respect to $\theta \in[-\pi, \pi]$ by a result of Baernstein [1, Corollary on p. 154].

On the other hand,

$$
g\left(r e^{i \theta},-a ; U\right)=\mathfrak{g}\left(r e^{i \theta}-a,-2 a\right)=\log \left|\frac{1+2 a\left(r e^{i \theta}-a\right)}{r e^{i \theta}+a}\right| .
$$

Using the Maple computer algebra package (one could presumably also do this by hand), we find that

$$
\frac{d g\left(r e^{i \theta},-a ; U\right)}{d \theta}=\frac{\left(-1+6 a^{2}+2 r^{2}-8 a^{4}-8 a^{2} r^{2}\right) a r \sin \theta}{\Delta},
$$

where

$$
\begin{aligned}
\Delta= & 4 a^{2} r^{2}-8 a^{2} r^{2} c^{2}-a^{2}-2 a r c+4 a^{3} r c+4 a^{4} \\
& -4 a r^{3} c-4 a^{6}+16 a^{4} r^{2} c^{2}-8 a^{4} r^{2}-4 a^{2} r^{4}-r^{2}
\end{aligned}
$$


and $c=\cos \theta$. Since $g\left(r e^{i \theta},-a ; U\right)$ is certainly not constant in $\theta$, it follows that $d g\left(r e^{i \theta},-a ; U\right) / d \theta$ can only vanish at $\theta=0$ and at $\theta=\pi$, so that

$$
\min _{\theta} g\left(r e^{i \theta},-a ; U\right)=\min (g(r,-a ; U), g(-r,-a ; U)) .
$$

But a completely elementary analysis of the explicit formulae (3) and (4) shows that for $0<a<\frac{1}{2}$ and $r$ sufficiently close to $a$ we have $g(-r,-a ; U)>g(-r, a ; U)$, while the inequality $a<r<1-a$ implies that $g(r,-a ; U)>g(-r, a ; U)$. By (5), the proof is complete. By a more precise but still elementary analysis it should in principle be possible to determine the exact range of values of $r$ for which the result holds.

Proof of the lemmas. We first give the common part of the two proofs. Let $U$ be $U_{a b c d}$ or $U_{a b}$, depending on which of the two examples we wish to work with. By symmetry in both cases we need only consider $\pi<\theta<\pi+\varepsilon$. Define $G(z)=$ $g(z, 0 ; U)$ and $G^{*}(z)=g\left(z, 0 ; U^{*}\right)$, and for $x \in(-1,1)$ let

$$
H(x)=\lim _{y \rightarrow 0+} \frac{G(x+i y)-G(x)}{y} \quad \text { and } \quad H^{*}(x)=\lim _{y \rightarrow 0+} \frac{G^{*}(x+i y)-G^{*}(x)}{y} .
$$

We shall later prove that if $U=U_{a b c d}$, then

$$
H(x)>H^{*}(-|x|) \text { for some } x \in(a, b) \cup(-d, c) .
$$

Assume this for now. In this case we let $r=|x|$. We may assume that $x \in(a, b)$, since the reader will easily see that the proof in the case of $x \in(-d,-c)$ would be quite analogous. On the other hand, in the case of $U=U_{a b}$ we shall show that

$$
H^{*}(x) \rightarrow 0 \text { as } x \downarrow-b .
$$

Again, assume this for now. But, it is easy to see that $\liminf _{r \uparrow b} H(r)>0$, so that we may choose $a^{\prime} \in(a, b)$ such that for every $r \in\left[a^{\prime}, b\right)$ we have $H^{*}(-r)<H(r)$. Fix such an $r$, then.

Thus, in either case we are working with an $r$ such that $H^{*}(-r)<H(r)$. Assuming this, the proof from now on will actually be the same in both of the cases. For $t \in[0, \pi]$, let $f(t)=G\left(r e^{i t}\right)$ and $g(t)=G^{*}\left(r e^{i(t+\pi)}\right)$. Then, from the inequality $H(r)>H^{*}(-r)$ together with the standard fact that in both of the cases under consideration we have $f^{\prime}(t) \rightarrow r H(r)$ and $g^{\prime}(t) \rightarrow r H^{*}(-r)$ as $t \rightarrow 0+$, it follows that there must then be an $\varepsilon_{1}>0$ such that $f^{\prime}(t)>g^{\prime}(t)$ whenever $0<t<\varepsilon_{1}$. Since the Green's function of a domain vanishes on the boundary, in both of our cases we easily see that $f(0)=g(0)=0$, and it follows that $f(t)>g(t)$ for $0<t<\varepsilon_{1}$.

Let $F$ be the non-decreasing rearrangement of $f$ on $[0, \pi]$, i.e., a non-decreasing function equimeasurable with $f$ on $[0, \pi]$. The positivity of $f^{\prime}$ near zero and the vanishing of $f$ at zero, together with the easy fact that in both of our cases $f$ is bounded away from zero on every interval $[\delta, \pi]$ where $\delta>0$, all imply that we may find an $\varepsilon_{2}>0$ such that $f(t)<f\left(t^{\prime}\right)$ whenever $0 \leq t<\varepsilon_{2}$ and $t<t^{\prime} \leq \pi$. Then, it follows that $f(t)=F(t)$ whenever $0 \leq t<\varepsilon_{2}$. Hence, $F(t)=f(t)>g(t)$ for $0<$ $t<\varepsilon$, where $\varepsilon=\min \left(\varepsilon_{1}, \varepsilon_{2}\right)$. By the symmetry of $U$ we have $F(t)=\tilde{G}\left(r e^{i(t+\pi)}\right)$, and the desired conclusions of the lemmas follow. 
It remains to prove (6) for $U=U_{a b c d}$ and (7) for $U=U_{a b}$. First let $U=U_{a b c d}$. Let $\mathfrak{g}$ be Green's function for the unit disc as in (2). By Green's formula, much as in [2, pp. 46-48] or [4, p. 105, eqn. (5.4)], we can see that for $z \in D \backslash\{0\}$ we have:

$$
G(z)=\log \frac{1}{|z|}-2 \int_{a}^{b} \mathfrak{g}(z, x) H(x) d x-2 \int_{c}^{d} \mathfrak{g}(z,-x) H(-x) d x
$$

and

$$
G^{*}(z)=\log \frac{1}{|z|}-2 \int_{a}^{b} \mathfrak{g}(z,-x) H^{*}(-x) d x-2 \int_{c}^{d} \mathfrak{g}(z,-x) H^{*}(-x) d x .
$$

Now in order to obtain a contradiction, suppose that we had $H(x) \leq H^{*}(-|x|)$ for every $x \in(-d,-c) \cup(a, b)$. By (9), we would have

$$
G^{*}(z) \leq \log \frac{1}{|z|}-2 \int_{a}^{b} \mathfrak{g}(z,-x) H(x) d x-2 \int_{c}^{d} \mathfrak{g}(z,-x) H(-x) d x .
$$

Now, fix $z \in(-d,-c)$. From the explicit formula for $\mathfrak{g}(z, w)$ one can verify that $\mathfrak{g}(z,-x)>\mathfrak{g}(z, x)$ whenever $x>0$ and $z<0$. Since $H$ is known to be positive on $(a, b)$, it would follow from (10) that

$$
G^{*}(z)<\log \frac{1}{|z|}-2 \int_{a}^{b} \mathfrak{g}(z, x) H(x) d x-2 \int_{c}^{d} \mathfrak{g}(z,-x) H(-x) d x .
$$

But by (8), the right-hand side is precisely $G(z)$ so that we would have $G^{*}(z)<$ $G(z)$. On the other hand, since both $G$ and $G^{*}$ vanish on $(-d,-c)$, we have $G(z)=G^{*}(z)=0$. We thus obtain a contradiction, and so (6) holds.

Now let $U=U_{a b}$. Note that $G^{*}$ is harmonic and bounded on the cone $C_{-b, \delta}=$ $\left\{-b+r e^{i \theta}: 0<r<\delta, 0<\theta<\pi / 2\right\}$, where $\delta$ is chosen sufficiently small so that the cone fits inside $U^{*}$ and $\delta<b-a$ so that $G^{*}$ vanishes on the two edges $[-b,-b+\delta]$ and $[-b,-b+i \delta]$ of the cone. (See Figure 3.)

Thus, (7) will clearly follow as soon as we can prove that whenever $h$ is a harmonic function on the translated and dilated cone $C=C_{0,1}=\left\{r e^{i \theta}: 0<r<1,0<\theta<\right.$ $\pi / 2\}$, with $h$ bounded on $C$ and vanishing on the two edges [0,1] and [0,i], then the normal derivative of $h$ at $x \in(0,1)$ tends to zero as $x \downarrow 0$. To prove this claim, note that

$$
R(z)=\left(\frac{1+z^{2}}{1-z^{2}}\right)^{2}
$$

is a univalent map of $C$ onto the upper half plane, with $R(0)=1$. Now, define $h_{1}=h \circ R^{-1}$ on the upper half plane. This will be a bounded harmonic function, vanishing on the interval $(0, \infty)$ since this interval is the image under $R$ of the edges $[0,1)$ and $[0, i)$ of $C$. It is easy then to see that the normal derivative of $h_{1}$ will have to be bounded by some finite constant $K$ on the interval $\left[\frac{1}{2}, \frac{3}{2}\right]$. Then, since $R$ is analytic in a neighbourhood of $x$ if $x \in(0,1)$, it follows that the normal derivative of $h=h_{1} \circ R$ for $x$ sufficiently close to 0 is bounded by $K\left|R^{\prime}(x)\right|$. But as $x \downarrow 0$, we see directly that $R^{\prime}(x) \rightarrow 0$, and so the normal derivative tends to zero as desired. This proves (7). 


\section{ACKNOWLEDGMENT}

The author would like to thank Professors Albert Baernstein II and Gregory F. Lawler for proofreading and providing helpful suggestions on improving the presentation of this paper. He would also like to thank Professor Baernstein for suggesting that a simply connected counterexample be constructed.

\section{REFERENCES}

1. Albert Baernstein II, Integral means, univalent functions and circular symmetrization, Acta Math. 133 (1974), 139-169. MR 54:5456

2. Arne Beurling, Études sur un problème de majoration, Thèse pour le doctorat, Almqvist \& Wiksell, Uppsala, 1933.

3. W. K. Hayman, Research Problems in Function Theory, Athlone Press, London, 1967. MR 36:359

4. Rolf Nevanlinna, Analytic Functions, Springer Verlag, New York, 1970. MR 43:5003

University of British Columbia, Vancouver, British Columbia, Canada V6T 1 Z2

E-mail address: pruss@math.ubc.ca 